\title{
TechNêts
}

\section{CRISPR/CAS9: THE GOLD STANDARD OF GENOME EDITING?}

\section{Alfie Gleeson \& Abigail Sawyer inyestigate whether a new generation of Cas enzymes can knock CRISPR/ Cas9 off its pedestal}

For reprint orders, please contact: reprints@futuremedicine.com

C RISPR/Cas9 has been at the heart of many major breakthroughs in genome editing. Since the first report of CRISPR/Cas9 in 2012, a search of the online research paper database, PubMed [1], shows scientists have published more than 5000 papers mentioning its name. However, it may now be time to move on and embrace the next generation of genome editing as the limitations of CRISPR/Cas9 become more apparent following its increased adoption for use by laboratories across the world.

Until recently, it has been extremely difficult to use CRISPR to edit DNA outside of cell lines, preventing CRISPR/Cas9 research moving onto clinical stages. The size of CRISPR/Cas9 has also inhibited its functionality; the enzyme itself is quite large, making targeted delivery of the enzyme difficult. Finally, we know it can edit DNA but can the system be modified to edit RNA too?

These are, no doubt, serious problems, but they are exactly the kind of problems scientists enjoy solving. The CRISPR system is rich with possibility, not just limited to Cas9. A whole family of enzymes exist within the system that, along with the fantastic research and experimental skills of top scientists all over the world, can be used to overcome these hurdles.

The application of these newly discovered CRISPR family members and variants of Cas enzymes is producing fantastic results and the CRISPR family is only getting bigger, more varied and more effective.

\section{Cas12a}

In order for CRISPR to fully realise its potential as an integral component in gene therapy it needs to be translated to the clinical phase. However, due to its restriction to cell lines, this has not previously been possible. Scientists at Christiana Care Health System's Gene Editing Institute (DE, USA) have made a breakthrough by successfully editing DNA in a test tube; marking the first time DNA has been edited by CRISPR outside of the cell [2].

The researchers hope this will enable them to quickly and precisely engineer multiple changes to a DNA code. It is believed that this new CRISPR technology could be utilized as a diagnostic tool, replicating genetic mutations found in the tumors of cancer patients.

The rapid identification of the correct mutation in an individual's tumor will allow a more targeted treatment strategy to be implemented, as mutations that cause cancer can differ from one person to the next.

"With this new advance, we should be able to work with laboratory cultures and accomplish gene edits in less than a day, significantly reducing the time required for diagnostics compared to other CRISPR tools, and with much greater precision," commented Eric Kmiec, the director of the Gene Editing Institute and lead author of the study. "This is particularly important for diagnostics linked to cancer care where time is critical."

Kmiec also explained that unlike other CRISPR tools that can only edit or repair shorter segments of DNA code within a gene, this new CRISPR tool could be utilized to remove and replace entire genes. This could enable CRISPR to treat diseases such as Alzheimer's, which appear to involve damage in multiple genes. The new treatment would involve gene replacement instead of gene editing. 


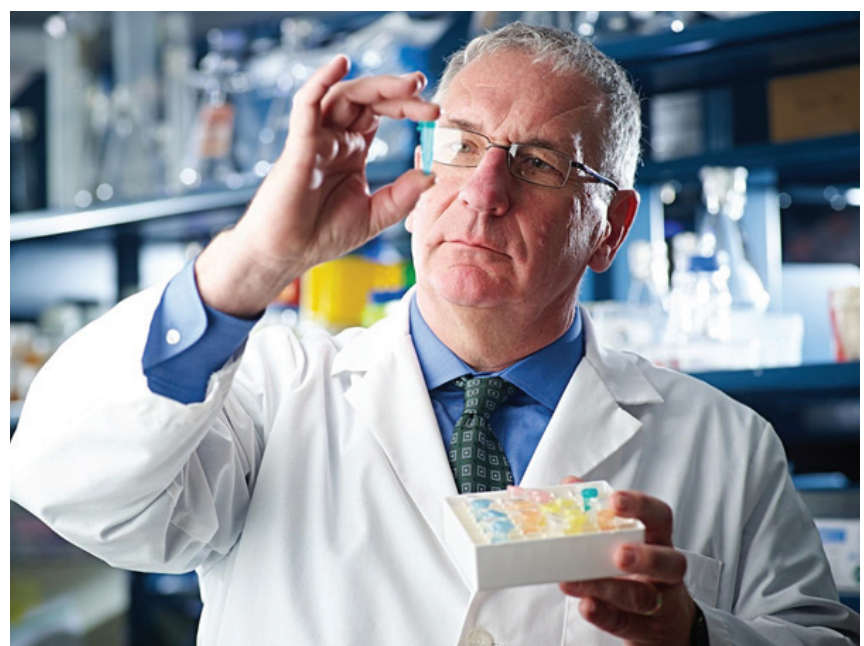

Eric Kmiec, director of The Gene Editing Institute, looks over in vitro samples of CRISPR in the lab.

Credit: The Gene Editing Institute.

The CRISPR/Cas9 system performed poorly when it was trialed by the research team in vitro, which prompted the team to turn to a new target. Genes modified by the team were contained in a DNA plasmid and utilized an enzyme referred to as Cas12a (also known as Cpf1).

"It could be that there is something within the very complex machinery of a cell that allows the Cas9 enzyme to more easily accomplish deletions and insertions," explained lead author Brett Sansbury.

"But it performed very poorly in our cell-free extracts."

Another benefit of Cas12a is that it produces DNA with sticky ends, unlike Cas9, which produces DNA with blunt ends. This allows for the easier insertion and attachment of a new code.

The team is now working to create a commercial product utilizing the technology for cancer diagnosis and believes that now they have brought the gene-editing process in vitro, they will be able to gain crucial insights regarding exactly how CRISPR works to modify genetic code.

This groundbreaking research has resulted in the ability to edit DNA in vitro, but what about editing in vivo?

\section{dCas9}

Researchers from the biomedical engineering department of Duke University (NC, USA) have utilized a CRISPR/Cas9 genetic engineering technique to silence a gene that regulates cholesterol concentrations in murine models [3]. The results of the experiment are promising for the clinical application of CRISPR as it is the first time this targeted therapy has been successfully delivered in animal models.

The gene that regulates cholesterol levels is PCSK9. Silencing the PCSK9 gene would have a greater effect on cholesterol than current drugs that target it. Instead of blocking the receptor, the CRISPR therapy would stop it being generated in the first place. The researchers had already seen success in cultured cells but wanted to take the therapy to the next level, as author Charles Gersbach explains:

"We previously used these same types of tools to turn genes on and off in cultured cells and we wanted to see if we could also deliver them to animal models with an approach that is relevant for gene therapy. We wanted to change the genes in a way that would have a therapeutic outcome, and PCSK9 is a useful proof-of-concept given its role regulating cholesterol levels, which in turn affect health issues like heart disease."

However, to administer the therapy the researchers had to repackage it and develop a new delivery system. For this they decided to use viral vectors. These viruses are great for targeting specific tissues for gene therapy trials but they only have a small cargo limit. Conventional Cas9 is too large so the team turned to alternatives. Conveniently there is a smaller variant of Cas9 that is derived from Streptococcus aureus, unlike the common variant, which is derived from Staphylococcus pyogenes.

Now the scientists had developed the Cas9 that suited their needs and the delivery system to get it to the target, they also wanted to modify the function. They only needed Cas 9 to bind to the target whilst another associated protein, KRAB, did the work of silencing the gene. So, the team deactivated the DNA slicing function, creating dead Cas9, also known as dCas9.

Despite the success of the therapy in silencing PCSK9, there was evidence of a slight immune response to the treatment. However coauthor Pratiksha Thakore thinks that understanding this response is the next step CRISPR therapies must take to translate to the clinic.

"Following injection, we saw that levels of our target gene, PCSKO, were reduced, but we also observed increases in expression of many immune cell genes, which indicates that immune cells were infiltrating the liver after we delivered Cas9 to the mice. Gaining a better understanding of this immune response and how to modulate it will be important for using Cas9 technologies for therapies."

The success of clinical treatment using CRISPR enzymes will rely on how the body responds to them. The variety in the bacterial CRISPR family generates many new possibilities but each protein will carry a different risk of immune response. Despite this looming hurdle Thakore is excited by the potential outcomes that can be generated by new experimentation and discovery.

"There are still lots of things for us to explore with this approach. CRISPR/Cas9 tools have worked so well in cell culture models that it's exciting to apply them more in vivo, especially when we're examining important therapeutic targets and using delivery vehicles that would be relevant to treating human diseases."

\section{CasX \& CasY}

We have seen that in very specific circumstances, CRISPR can edit DNA outside of the cell both in vitro and in vivo. However, the research by Thakore et al. would not have been possible without the use of the smaller Cas 9 variant.However, even smaller and more compact CRISPR enzymes have been discovered.

They have been labelled as the most compact CRISPR systems yet. Researchers from the University of California, Berkeley (CA, USA) published their discovery of the new Cas enzymes from uncultivated microbes, Cas $\mathrm{X}$ and Cas $\mathrm{Y}$, in 2017 [4]. 


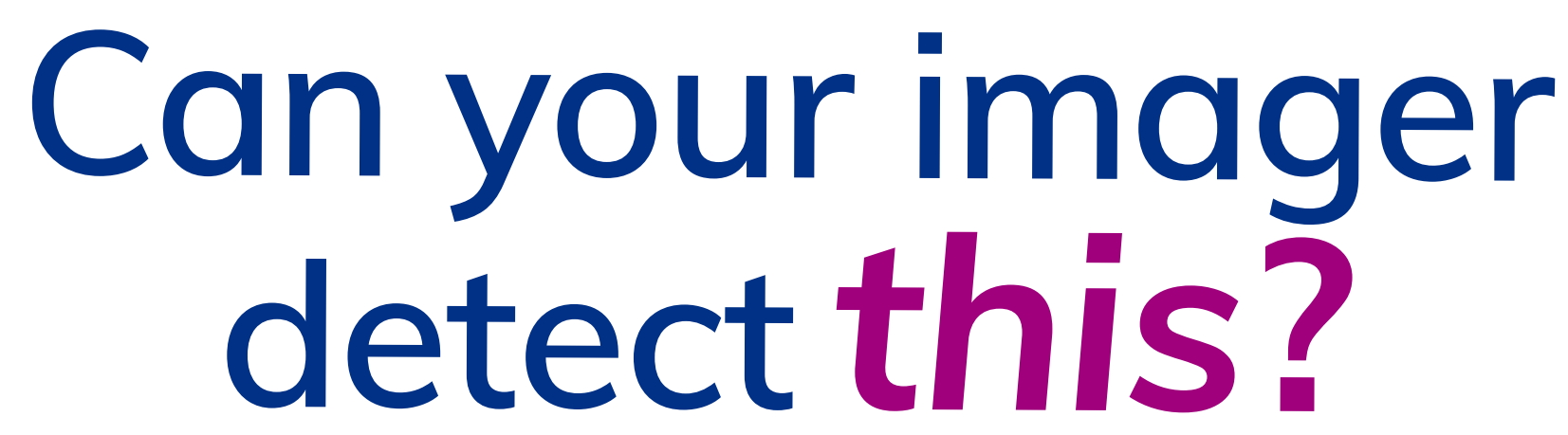

\begin{tabular}{ll|}
\multicolumn{2}{c|}{ Visible Fluorescence } \\
\hline $371 \mathrm{pg}$ & $0.72 \mathrm{pg}$ \\
\hline & \\
Sample & AzureSpectra 490 labeled \\
purified BSA & 488 \\
Imaging & 488 \\
LOD & $0.72 \mathrm{pg}$ \\
DR & 6.3 \\
Linearity & $\mathrm{R}^{2}=0.99$ \\
\hline
\end{tabular}

\begin{tabular}{ll|}
\multicolumn{3}{c|}{ NIR Fluorescence } \\
\hline $1484 \mathrm{pg}$ \\
\hline Sample & $\begin{array}{l}\text { AzureSpectra } 550 \text { labeled } \\
\text { purified transferrin }\end{array}$ \\
Imaging & 520 \\
LOD & $2.9 \mathrm{pg}$ \\
DR & 5.7 \\
Linearity & $\mathrm{R}^{2}=0.992$
\end{tabular}

\section{Chemiluminescence}

\begin{tabular}{|ll|}
\hline $305 \mathrm{fg}$ & \\
\hline & \\
& \\
\hline Sample & Azure Radiance Substrate \\
Imaging & Chemiluminescence mode \\
LOD & $\begin{array}{l}\text { 305 fg Transferrin } \\
\text { (Western blot) }\end{array}$ \\
DR & 4.8 \\
Linearity & $\mathrm{R}^{2}=0.99$ \\
\hline
\end{tabular}

\section{Phosphor Imaging}
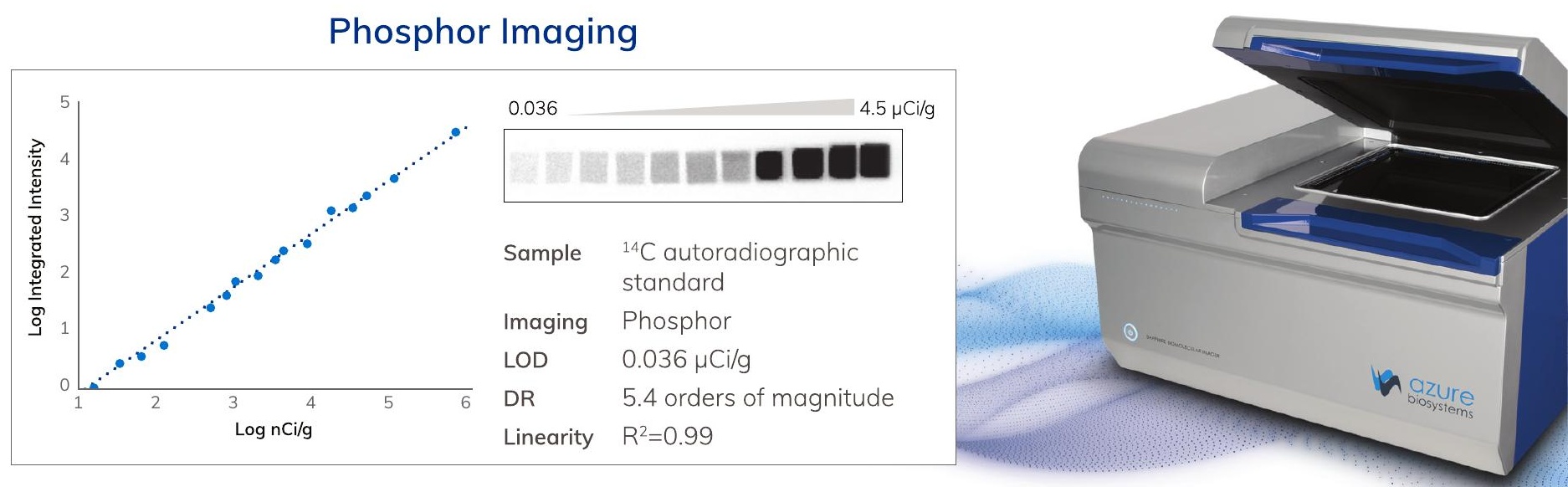

Find out just how low you can go! With sensitive detection and a wide dynamic range the Sapphire ${ }^{T M}$ Biomolecular Imager delivers the ultimate in imaging flexibility and performance. Learn more at azurebiosystems.com/sensitive-detection

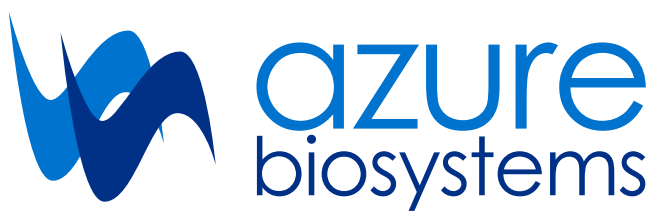


a

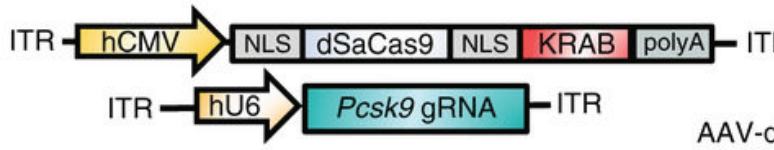

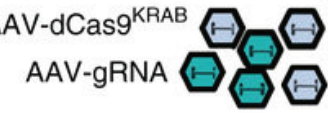
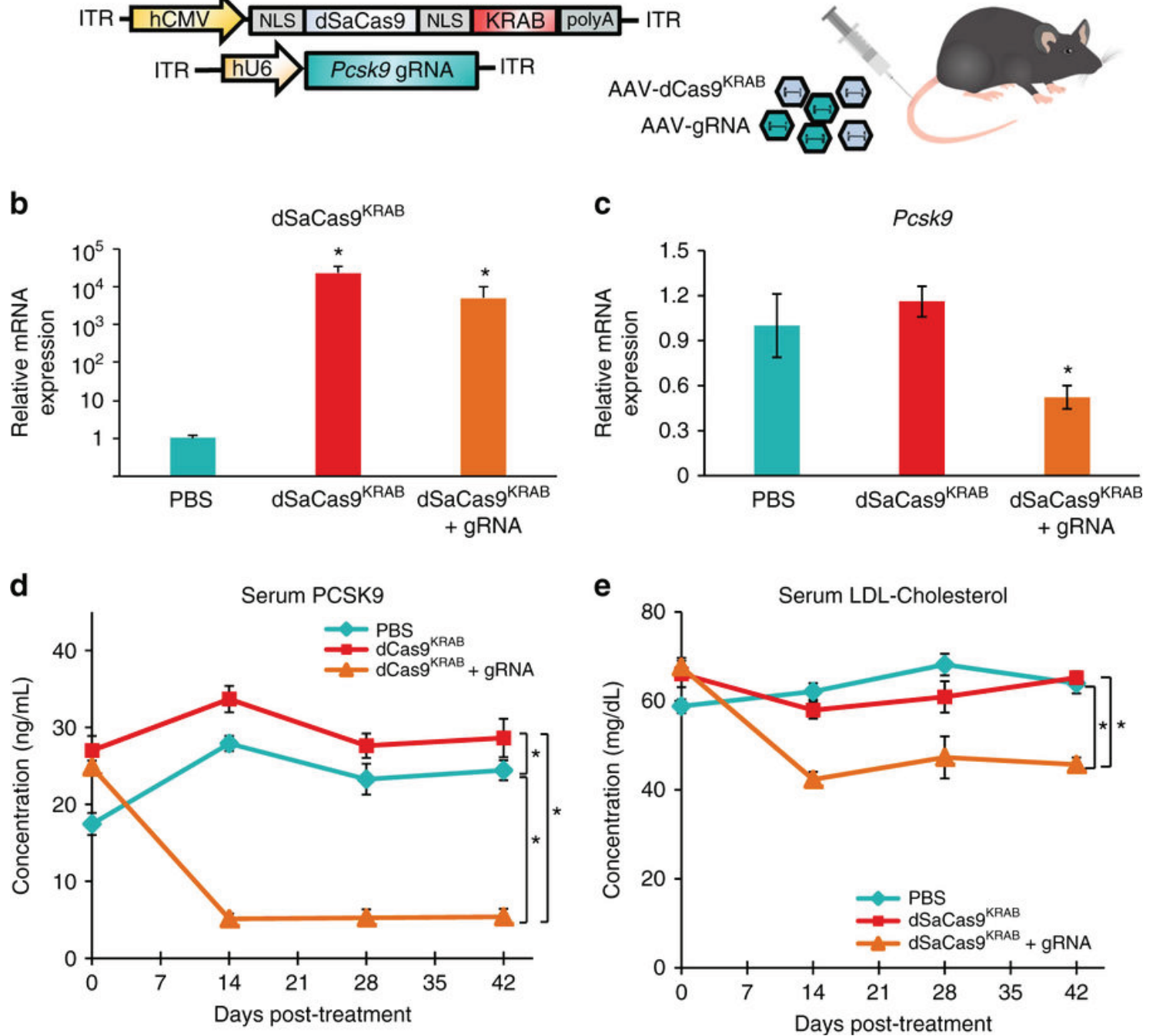

Targeted gene silencing in adult wild-type mice with Staphylococcus aureus dCas9 ${ }^{\mathrm{KRAB}}$.

(A) A dual adeno-associated virus (AAV) vector system was designed to deliver dSaCas9 ${ }^{\mathrm{KAB}}$ and PCSK9-targeting gRNA to adult wild-type mice via tail-vein injection. qRT-PCR for (B) dSaCas9 ${ }^{\mathrm{KRAB}}$ and (C) Pcsk9 expression was performed on mRNA from livers harvested from treated mice at 6 weeks post-injection (mean \pm s.e.m, $n=4$ mice). $p<0.05$ is indicated by *, determined by Student's t-test compared to controls. Serial serum collections were assayed for secreted (D) PCSK9 protein levels and e low-density lipoprotein cholesterol (mean \pm s.e.m., $n=4$ mice, ${ }^{\star} p<0.05$ by mixed design ANOVA with Tukey's post-hoc analysis). Reproduced with permission from [3].

It is notoriously difficult to deliver Cas proteins into cells for gene editing because the number of amino acids make them so large. However, CasX comprises 980 amino acids, in comparison to Cas9, which comprises 1368. This provides a potential advantage over the other Cas proteins.

"This is important biotechnologically, because if you look at it from the angle of genome editing, the delivery of small genes into cells is much easier than the delivery of large genes," explained Rotem Sorek of the Weizmann Institute of Science (Rehovot, Israel).

The team scanned metagenome databases they had built up over 15 years in search for gene sequences that are similar to those that code for Cas9. They found only two - CasX and CasY.

"These are particularly interesting because the key protein in these CRISPR systems is approximately the same as
Cas9, but is not Cas9. It is part of a minimal system that has obvious potential for gene editing," commented contributing author, Jill Banfield.

The researchers found these CRISPR systems in bacteria from deep underground at Crystal Geyser in California (USA) as well as in phyla living in groundwater. They were previously unexplored as they cannot be cultivated in the laboratory. This is because they are symbionts and rely upon other microbes for the nutrients needed to survive; however, this has opened a whole new path of exploration for microbes.

The team's hope is that as the database of uncultivated bacteria continues to expand, they will find yet more variants of the CRISPR/Cas9 system, as well as proteins with unusual functions that may be used in various applications. 
a

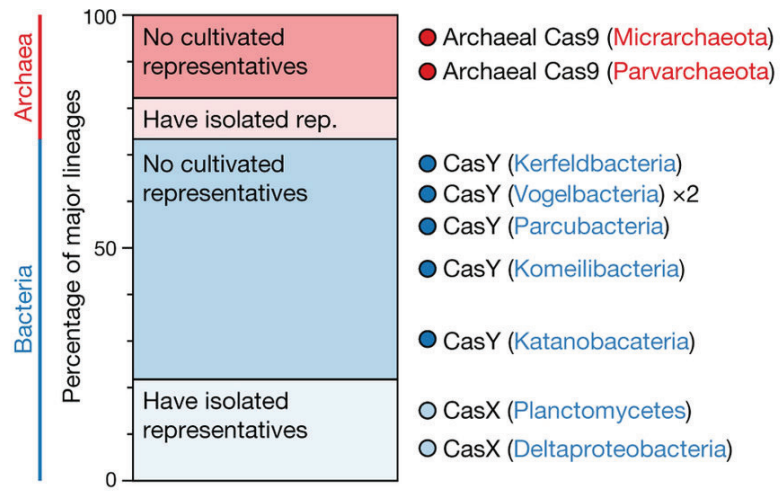

b

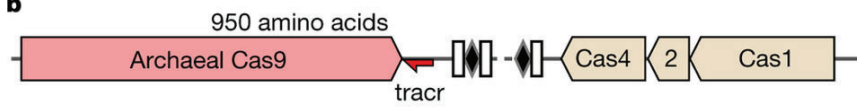

980 amino acids

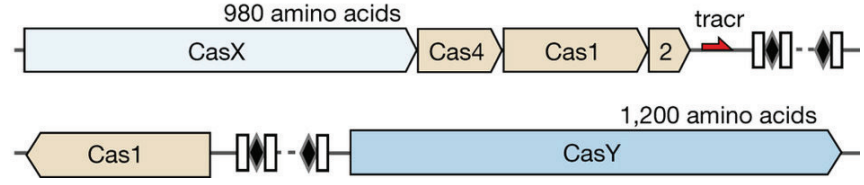

The location of Cas proteins. (a) Percentage of lineages with and without isolated representatives in Bacteria and Archaea, based on 31 major lineages described previously. The results highlight the massive scale of as-yet little-investigated biology in these domains. Archaeal Cas9 and the novel CRISPR-CasY were found exclusively in lineages with no isolated representatives. (b) Locus organization of the discovered CRISPR-Cas systems. Reproduced with permission from [4].

\section{CasRx}

Now we have seen how the extended CRISPR family can move DNA editing on from experimental stages to clinical trials, it is time to turn our attention to a new target.

The limitations in knowledge as to what extent mutations in DNA cause disease have led to a new class of Cas enzymes designed to target RNA as opposed to DNA, as the issues can be more easily pinpointed in RNA.

Scientists at the Salk Institute (CA, USA) have produced a new gene-editing technology to cut RNA. The tool, named CasRx, comes from the same family of CRISPR enzymes as Cas9 but has expanded the gene editing tool box from DNA to RNA [5]. In an experiment the team were able to correct a protein imbalance in a neuron exhibiting frontotemporal dementia (FTD).

CRISPR has been considered revolutionary in developing tools to treat genetic or gene-related disease. However, there are some diseases that can occur due to toxic or improperly spliced RNA, such as FTD. If a strand of DNA can be targeted then it follows that RNA can be too.
Author Silvana Konermann explains: "We began the project with the hypothesis that different CRISPR systems may have been specialized throughout an evolutionary arms race between bacteria and their viruses, potentially giving them the ability to target viral RNA."

To search for these potential CRISPR systems, the team utilized a bacterial DNA database. They identified the enzymes that target RNA and screened them to find the ones that would work best in human cells. They then put them into action to properly test the enzymes.

"Once we engineered CasRx to work well in human cells, we really wanted to put it through its paces," commented Konermann.

The team chose to experiment on neurons taken from a patient suffering with FTD. In this neurodegenerative disorder the ratio of the alternative versions of the tau protein are unbalanced. CasRx was delivered into the cells via a viral vector. It targeted the overabundant version of tau protein, restoring balance with $80 \%$ effectiveness.

Co-author Patrick Hsu is hopeful the new technology, adapted from nature, will expand the genetic tool box and enable the development of more therapies for disease:

"Nature is full of so many secrets, it's really a rich, untapped resource for inventing new technologies."

\section{Back to Cas9}

There may now be a wave of excitement initiated by this potential second generation of genome editing, but only time will tell if we really should move on from CRISPR/Cas9.

It will take many more years of work for these new Cas enzymes to find their way into clinical settings. When and if this happens, we will know if CRISPR/Cas9 really is the golden standard of genome editing or if the enthusiasm surrounding its discovery has overshadowed systems that are better suited for individual applications.

\section{References}

1. PubMed. www.ncbi.nlm.nih.gov/pubmed

2. Sansbury BM, Wagner AM, Nitzan E, Gabi T, Kmeic EB. CRISPR-directed in vitro gene editing of plasmid DNA catalyzed by Cpf1 (Cas12a) nuclease and a mammalian cell-free extract. CRISPR J. 1, 2 doi:10.1089/crispr.2018.0006 (2018).

3. Thakore PI, Kwon JB, Nelson CE et al. RNA-guided transcriptional silencing in vivo with S. aureus CRISPR-Cas9 repressors. Nat. Commun. doi:10.1038/ s41467-018-04048-4 (2018).

4. Burstein D, Harrington LB, Strutt SC et al. New CRISPR-Cas systems from uncultivated microbes. Nature 542, 237-241 (2017).

5. Konermann S, Lotfy P, Brideau NJ, Oki J, Shokhirev MN, Hsu PD. transcriptome engineering with RNA-targeting type VI-D CRISPR effectors. Cell doi:10.1016/j.cell.2018.02.033 (2018). 


\section{Powerful next-generation gene activation with Edit- $R^{\text {TM }}$ CRISPRa reagents}

Increase target gene expression in your cell lines with Dharmacon Edit-R CRISPR activation (CRISPRa) reagents. From one gene up to the whole human genome, our reagents make gain-of-function studies easy and efficient.

\section{Synthetic crRNA | Lentiviral sgRNA | Arrayed libraries}

\section{All Edit-R CRISPRa guide RNA formats provide effective target gene activation}

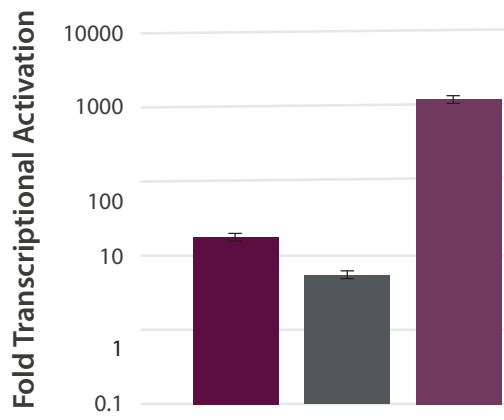

crRNA:tracRNA
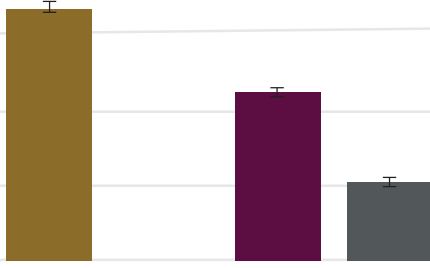

sgRNA particles
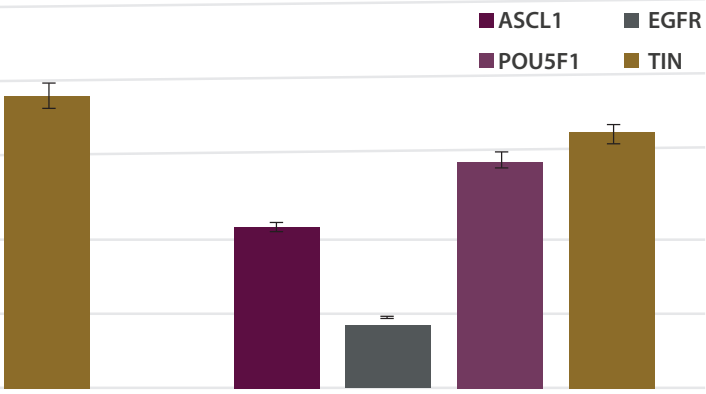

sgRNA plasmid

Edit-R CRISPRa guide RNA

U2OS cells stably expressing integrated Edit-R dCas9-VPR were treated with three formats of Edit-R CRISPRa guide RNAs: synthetic crRNA:tracrRNA, lentiviral sgRNA particles, or sgRNA plasmid, each targeting ASCL1, EGFP, POU5F1, or TTN genes. Relative gene expression was calculated using qRT-PCR. 